\title{
Association of Chinese Herbal Medicines Use with Development of Chronic Obstructive Pulmonary Disease Among Patients with Rheumatoid Arthritis: A Population-Based Cohort Study
}

This article was published in the following Dove Press journal:

International Journal of Chronic Obstructive Pulmonary Disease

\author{
Miao-Chiu Lin ${ }^{1} *$ \\ Hanoch Livneh (iD) ${ }^{2, *}$ \\ Wei-Jen $\mathrm{Chen}^{3, *}$ \\ Ning-Sheng $\mathrm{Lai}^{4,5}$ \\ Ming-Chi Lu ${ }^{4,5}$ \\ Tzung-Yi Tsai ${ }^{6-8}$ \\ 'Department of Nursing, Dalin Tzuchi \\ Hospital, The Buddhist Tzuchi Medical \\ Foundation, Chiayi 62247, Taiwan; \\ ${ }^{2}$ Rehabilitation Counseling Program, \\ Portland State University, Portland, OR \\ 97207-075I, USA; ${ }^{3}$ Department of Chinese \\ Medicine, Dalin Tzuchi Hospital, The \\ Buddhist Tzuchi Medical Foundation, Chiayi \\ 62247, Taiwan; ${ }^{4}$ Division of Allergy, \\ Immunology and Rheumatology, Dalin \\ Tzuchi Hospital, The Buddhist Tzuchi \\ Medical Foundation, Chiayi 62247, Taiwan; \\ ${ }^{5}$ School of Medicine, Tzu Chi University, \\ Hualien 97004, Taiwan; ' ${ }^{6}$ Department of \\ Environmental and Occupational Health, \\ College of Medicine, National Cheng Kung \\ University, Tainan 70428, Taiwan; \\ ${ }^{7}$ Department of Nursing, Tzu Chi \\ University of Science and Technology, \\ Hualien 62247, Taiwan; ${ }^{8}$ Department of \\ Medical Research, Dalin Tzuchi Hospital, \\ The Buddhist Tzuchi Medical Foundation, \\ Chiayi 62247, Taiwan \\ *These authors contributed equally to this \\ work
}

Correspondence: Ming-Chi Lu; Tzung-Yi Tsai

Tel +886-5-2648000-87। 3;

$+886-5-2648000-3209$

Fax +886-5-2648006

Email dm252940@tzuchi.com.tw;

dm732024@tzuchi.com.tw
Purpose: Rheumatoid arthritis (RA) patients appear to report a higher risk of chronic obstructive pulmonary disease (COPD). While Chinese herbal medicine (CHMs) is proven to lower COPD risk, the scientific evidence regarding its effect in relation to COPD onset among them is limited. This longitudinal cohort study aimed to determine the relationship between CHMs use and the COPD risk in RA patients.

Methods: Using the nationwide claim data, 8349 patients newly diagnosed with RA and simultaneously free of COPD between 1998 and 2010 were eligible for enrollment. From this sample, we enrolled 3360 CHMs users and 3360 non-CHMs users, randomly selected using propensity scores matching from the remaining cases. They were followed until the end of 2012 to record COPD incidence. The hazard ratio (HR) of COPD with regard to CHMs use was estimated by the Cox proportional hazards regression model.

Results: In the follow-up period, $136 \mathrm{CHMs}$ users and 202 non-CHMs users developed COPD, representing incidence rates of 5.16 and 7.66, respectively, per 1000 person-years. CHMs use was associated with a 32\% lower subsequent risk of COPD (adjusted HR: 0.68, 95\% Confidence Interval: 0.54-0.84). Eight commonly prescribed CHMs were discovered to be associated with lower COPD risk: Yan Hu Suo, Sāng Zhī, Dang Shen, Huang Qin, Jia-Wei -Xiao-Yao-San, Shu-Jing-Huo-Xue-Tang, Du-Huo-Ji-Sheng-Tang and Ge-Gen-Tang.

Conclusion: A significant association of CHMs use with a lower risk of COPD onset in RA patients was found, suggesting that $\mathrm{CHMs}$ could be integrated into conventional therapy to reduce COPD risk.

Keywords: rheumatoid arthritis, Chinese herbal medicines, chronic obstructive pulmonary disease, cohort study

\section{Introduction}

Rheumatoid arthritis (RA) is a chronic systemic inflammatory disorder affecting about $1 \%$ of the population worldwide, with many patients ultimately developing progressive functional limitations and physical disabilities. ${ }^{1}$ Notably, it usually occurs in middle-age adults, and more than one-third of affected individuals exhibit arthritisattributable work limitations, thus posing a significant burden for patients, families, and social care systems. ${ }^{2}$ In the United States, a recent investigation revealed that the healthcare cost of RA was approximately US\$20,919 per affected person per year, approximately three times higher than for non-RA patients. ${ }^{3}$ A review of the financial 
burden of RA in the US reported that the total annual costs for RA was US\$19.3 billion, and when adding the intangi-

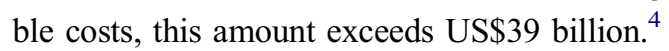

RA not only results in enormous economic losses but also presents a significant problem for public health. Due to the systemic inflammation inside the body, individuals with RA have a higher risk of pulmonary disease, especially chronic obstructive pulmonary disease (COPD). A recent British study following 24,625 patients with $\mathrm{RA}$, for over 10 years, found that those with RA had a $47 \%$ higher risk of COPD than a non-RA patient group. ${ }^{5}$ Another meta-analysis of four studies showed that RA patients indeed had a greater risk of COPD than did non-RA patients, with a pooled risk of 1.99 (95\% Confidence Interval [CI]: 1.61-2.45). ${ }^{6}$ Furthermore, a study revealed that patients with RA, suffering from concomitant COPD, had double the likelihood of mortality of those with RA only. ${ }^{7}$ Accordingly, RA patients were found to have 6-7 years' lower life expectancy as compared with the general population. ${ }^{8}$ These data, therefore, imply that it is of utmost urgency to prevent or treat COPD when managing RA subjects.

Recently, Chinese herbal medicines (CHMs) have been widely used in clinical practice to treat patients with chronic diseases. The estimated prevalence of CHMs usage varies across populations, ranging from $16 \%$ to $90 \%{ }^{9-12} \mathrm{CHMs}$ facilitate improved clinical outcomes and promote improved quality of life in individuals with chronic illnesses. A 10-year cohort study of 729 patients with advanced breast cancer showed that those receiving CHMs had a $45 \%$ reduced risk of all-cause mortality than those who did not receive CHMs. ${ }^{13}$ A randomized controlled trial of 352 individuals with COPD compared the effectiveness of combining conventional Western medicine with herbal products for 1 year. The results suggested that the integration of CHMs and Western medicine significantly improved pulmonary function, quality of life and psychological health (mood and depression) in this population. ${ }^{14}$

Although CHMs have attracted attention for some time, to the best of our knowledge, no study has been done to verify its long-term effect among RA patients, let alone the prevention of COPD in this group. From a standpoint of disease management for RA, the identification of the association of CHMs with lower COPD risk may help improve clinical outcomes and extend the life expectancy of RA patients. We, therefore, analyzed a nationwide populationbased database to assess COPD risk among RA patients who had either received or not received CHMs.

\section{Methods}

\section{Data Source}

For this study, we used a publicly released cohort dataset, the Longitudinal Health Insurance Database (LHID), comprised of approximately $1,000,000$ randomly sampled people, and collected all records from 1996 to 2012. The database has been confirmed by the National Health Research Institute to be representative of the Taiwanese population and its data have been used in many published scientific papers. ${ }^{15}$ The encrypted information protects patient privacy and allows linkage of all claims for the same patient within the database. This database contains all National Health Insurance (NHI) enrolment files, claims data and the registry for prescription drugs, to provide comprehensive utilization information on those individuals covered by the insurance program. This study was conducted in accordance with the Helsinki Declaration, and was evaluated and approved by the local Institutional Review Board and ethics committee of Buddhist Dalin Tzu Chi Hospital, Taiwan (No. B10004021-3).

\section{Study Subjects}

Diagnoses in the insurance claims data were coded using the International Classification of Disease, Ninth Revision, Clinical Modification (ICD-9-CM). We identified the patients who were aged 20 years or older and who sought ambulatory health care services between 1998 and 2010 for RA (ICD-9-CM code 714.0). To reduce the potential for disease misclassification, only those with catastrophic illness certification due to RA were recruited. In Taiwan, insured persons with major diseases, such as schizophrenia, mood disorders, immune disease and cancer, can apply for a catastrophic illness certificate that grants exemption from co-payment. The date when each RA patient gained approval for catastrophic illness registration was considered as the index date. To confirm that all patients with RA were indeed incident cases, only new-onset RA cases were included $(\mathrm{n}=8725)$. Therefore, the 312 patients diagnosed with COPD before the date of the first RA diagnosis were excluded from the study. Patients were considered to have a history of COPD if they had at least three outpatient visits or at least one inpatient claim for COPD (ICD-9-CM codes 491, 492 and 496), dating from 1996, when the computerized claims data from the LHID became available, until the date of the cohort study. Also excluded were those with missing data and those who were not followed for at least 
1 year after RA onset ( $\mathrm{n}=64)$. Overall, we identified 8349 new-onset RA subjects (Figure 1).

Thereafter, we applied the frequency of visits to Chinese medicine physicians to confirm the CHMs exposure of each RA patient. Those who used CHMs for more than 30 days after RA onset were considered CHMs users; enrollees treated for 30 days or less were considered non-CHMs users. ${ }^{16}$ Using this procedure, 3360 cases were designated as CHMs users. A comparison cohort was randomly selected from the remaining insured RA cases without $\mathrm{CHMs}$ use. For each RA patient receiving $\mathrm{CHMs}$ treatment, one control patient not receiving CHMs treatment was selected by 1:1 matching based on a propensity score. Propensity scores representing the likelihood of receiving CHMs were calculated using logistic regression analysis, conditional on the baseline covariates listed in Table 1. Person-years (PYs) of the nonCHMs users were determined by calculating the time from the index date to the earliest of one of the following: the diagnosis of COPD, the date of withdrawal from the insurance program or the date of December 31, 2012. The PYs of CHMs users was calculated from the initiation of CHMs in combination corrected by immortal time bias.

8725 patients aged $\geq 20$ years with newly diagnosed

RA treated between 1998 and 2010

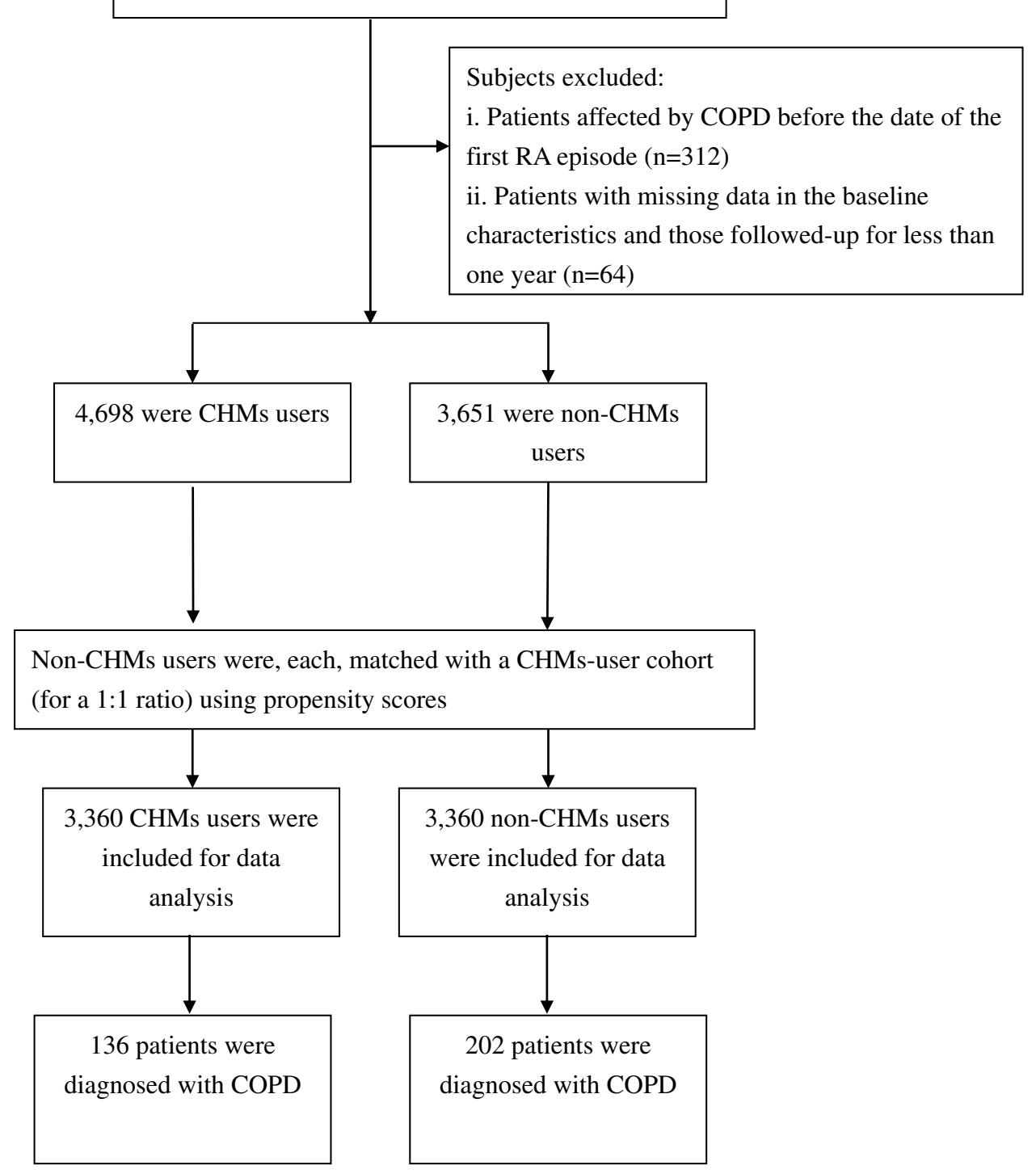

Figure I Flowchart showing the method of selecting and following study subjects. 
Table I Characteristics of the Study Subjects

\begin{tabular}{|c|c|c|c|c|}
\hline \multirow[t]{2}{*}{ Variables } & \multirow[t]{2}{*}{ Total Group } & \multirow{2}{*}{$\begin{array}{l}\text { Non-CHMs Users } \\
\mathrm{N}=3360 \text { (\%) }\end{array}$} & \multirow{2}{*}{$\begin{array}{l}\text { CHMs Users } \\
N=3360 \text { (\%) }\end{array}$} & \multirow[t]{2}{*}{$P$} \\
\hline & & & & \\
\hline Age (years) & & & & 0.75 \\
\hline$\leq 50$ & $2743(40.8)$ & $\mid 378(4 \mid .0)$ & $1365(40.6)$ & \\
\hline$>50$ & $3977(59.2)$ & 1982(59.0) & 1995(59.4) & \\
\hline Mean (SD) & $53.8 \pm 14.0$ & $53.80 \pm 14.3$ & $53.72 \pm 13.5$ & 0.81 \\
\hline Sex & & & & 0.53 \\
\hline Female & $4945(73.6)$ & $246 \mid(73.2)$ & 2484(73.9) & \\
\hline Male & $1775(26.4)$ & $899(26.8)$ & $876(26.1)$ & \\
\hline Monthly income & & & & 0.44 \\
\hline Low & $2972(44.2)$ & I504(44.8) & I468(43.7) & \\
\hline Median & $3485(51.9)$ & $1733(5 \mid .6)$ & $1752(52.1)$ & \\
\hline High & 263(3.9) & $123(3.7)$ & $140(4.2)$ & \\
\hline Residential area & & & & 0.84 \\
\hline Urban & $3842(57.2)$ & 1933(57.5) & $1909(56.8)$ & \\
\hline Suburban & $1043(15.5)$ & $518(15.4)$ & $525(15.6)$ & \\
\hline Rural & $1835(27.3)$ & $909(27.1)$ & $926(27.6)$ & \\
\hline Medication use & & & & 0.50 \\
\hline Yes & $5008(74.5)$ & $2492(74.2)$ & $2516(74.9)$ & \\
\hline No & $17 \mid 2(25.5)$ & $868(25.8)$ & $844(25.1)$ & \\
\hline \multicolumn{5}{|l|}{ Comorbidity } \\
\hline Hypertension & $1816(27.0)$ & $902(26.8)$ & $914(27.2)$ & 0.74 \\
\hline Diabetes & $850(12.6)$ & $4 I I(I 2.2)$ & $439(13.1)$ & 0.30 \\
\hline Heart disease & $1038(15.4)$ & $499(14.9)$ & $539(16.0)$ & 0.18 \\
\hline Chronic kidney disease & $87(1.3)$ & $49(1.5)$ & $38(1.1)$ & 0.24 \\
\hline Cancer & $206(3.1)$ & $102(3.0)$ & $104(3.1)$ & 0.89 \\
\hline Alcohol dependence syndrome & $14(0.2)$ & $7(0.2)$ & $7(0.2)$ & 0.99 \\
\hline Tobacco use & $7(0.1)$ & $5(0.1)$ & $2(0.1)$ & 0.26 \\
\hline Follow-up time (years) (mean, median) & 7.84(7.57) & $7.85(7.56)$ & 7.84(7.59) & \\
\hline
\end{tabular}

\section{Covariate Assessment}

Sociodemographic factors considered in this study included age, sex, income for estimating insurance payment, and urbanization level of the subject's residential area. Monthly incomes were stratified into three levels: $\leq$ New Taiwan Dollar (NTD) \$17,880, NTD\$17,881-43,900 and $\geq$ NTD\$43,901. Urbanization levels were divided into three strata: urban (levels 1-2), suburban (levels 3-4) and rural (levels 5-7) areas. Level 1 refers to the "most urbanized" and level 7 refers to the "least urbanized" communities. ${ }^{17}$ Baseline comorbidities included hypertension (ICD-9-CM codes 401-405), diabetes (ICD-9-CM code 250), heart disease (ICD-9-CM codes 410-429), chronic kidney disease (ICD-9-CM code 585), cancer (ICD-9-CM codes 140-208), alcohol dependence syndrome (ICD-9-CM code 303) and tobacco use (ICD-9-CM code 305.1); all these data were based on individual medical records 1 year prior to initial entry into the cohort. In addition, medication usage was stratified into whether or not the patient received corticosteroids or disease-modifying antirheumatic drugs for more than 6 months after the index date.

\section{Statistical Analysis}

We performed $\chi^{2}$ test and $t$-test to examine the differences in demographic characteristics and comorbidities between RA patients with and without CHMs treatment. Then, the incidence rate of COPD between the two groups was calculated as the number of cases per 1000 PYs. Cox proportional hazards regression analysis was then applied to compute the HR with $95 \%$ CI of COPD risk in association with CHMs use. To further test the robustness of the relationship between CHMs use and COPD risk, we divided the CHMs 
users into three subgroups: those who used CHMs for 31-365 days, those who used CHMs for 366-730 days and those who used CHMs for more than 730 days. We also used the Kaplan-Meier method to estimate the cumulative risk of COPD between groups and tested the difference with the log-rank test. Furthermore, a stratified analysis by age and sex using Cox proportional hazards regression was conducted to assess the HR of COPD among the subjects who did and did not receive CHMs. $\log (-\log [$ survival] $)$ versus $\log$ of survival time plot was inspected to verify the proportional hazards assumption. All analyses were conducted using SAS version 9.3 software (SAS Institute Inc, Cary, NC, USA). Differences of $P<0.05$ were considered statistically significant.

\section{Results}

The CHMs user and non-CHMs user cohorts provided data for 3360 subjects each, with a mean duration of 7.85 and 7.84 years, respectively. After the matching procedure with propensity score, there was no significant difference between the two groups in age, sex, monthly income, residential area and comorbidities, indicating that the two groups were comparable in terms of these characteristics (Table 1).

Among all eligible RA subjects, 338 first episodes of COPD occurred, 202 in non-CHMs users and 136 in CHMs users, during follow-up periods of 26,380.64 and 26,352.11 PYs, respectively. The incidence rate of COPD was significantly lower in CHMs users than in non-CHMs users (5.16 vs 7.66, respectively, per 1000 PYs), with an adjusted HR of 0.68 (95\% CI: $0.54-0.84$ ) (Table 2). Of note, those who used CHMs for more than 730 days had reduced the risk of COPD by $72 \%$. Results of the Kaplan-Meier survival curve and log-rank tests also supported a statistically significant difference in the survival rate free from COPD across the three groups of users during the follow-up period. Those receiving CHMs for more than 730 days had a significantly lower incidence rate of COPD than those not receiving CHMs $(P<0.001)$ (Figure 2).
Table 3 presents the results from the analysis stratified by age and sex. Collectively, a more significant beneficial effect of CHMs was observed among older subjects. Furthermore, multivariable stratified analysis verified that the benefit of CHMs therapy in reducing the incidence of COPD was more predominant in females, with an adjusted HR of 0.57 (95\% CI: 0.42-0.77) (Table 3). The 10 most commonly prescribed herbal formulae for those with RA are summarized in Table 4. Among them, the prescriptions of Yan Hu Suo, Sāng Zhī, Dang Shen, Huang Qin, Jia-Wei -Xiao-Yao-San, Shu-Jing-Huo-Xue-Tang, Du-Huo-JiSheng-Tang and Ge-Gen-Tang were associated with a decreased risk of COPD (Table 4).

\section{Discussion}

This is the first evidence-based cohort study addressing the association between CHMs use and COPD risk in patients with RA using a large nationwide claims-based data source. In this follow-up study of 15 years (1998-2012), we found that patients with RA who were receiving CHMs had a 32\% lower likelihood of COPD than those not using CHMs. Furthermore, those receiving CHMs for more than 2 years were found to have a nearly $72 \%$ lower risk of having COPD. The dose-response relationship may elucidate the causal relationships between CHMs use and the decrease in the predisposition to develop COPD. No previous studies have been conducted to determine the longtern impact of CHMs use on COPD risk among RA patients, thus rendering a comparison of results impossible. But the positive therapeutic effect of CHMs observed in this study is consistent with earlier reports and adds to the growing body of literature on this topic. ${ }^{12,18}$

Additionally, findings from our study indicated that older patients benefited the most, by receiving $\mathrm{CHMs}$ treatment, from lowering the risk of COPD, echoing the findings of an earlier report. ${ }^{19}$ Previous research indicated that younger adults are more likely than older adults to gain prompt access to social resources and maintain close

Table 2 Risk of COPD for RA Patients with and Without CHMs Use

\begin{tabular}{|l|l|l|l|l|l|l|}
\hline Patient Group & N & Events & PYs & Incidence & Crude HR (95\% Cl) & Adjusted HR* (95\% Cl) \\
\hline Non-CHMs users & 3360 & 202 & $26,380.64$ & 7.66 & 1.00 & 1 \\
CHMs users & 3360 & 136 & $26,352.11$ & 5.16 & $0.67(0.54-0.83)$ & $0.68(0.54-0.84)$ \\
CHMs use for 3I-365 days & 2749 & 122 & $20,773.28$ & 5.87 & $0.77(0.60-0.94)$ & $0.77(0.60-0.93)$ \\
CHMs use for 366-730 days & 346 & 9 & 2966.02 & 3.03 & $0.40(0.20-0.77)$ & $0.44(0.23-0.80)$ \\
CHMs use for more than 730 days & 265 & 5 & $2612.8 \mathrm{I}$ & 1.91 & $0.25(0.11-0.63)$ & $0.28(0.11-0.67)$ \\
\hline
\end{tabular}

Notes: *Model adjusted for sex, age, urbanization level, monthly income, medication use and comorbidities. 


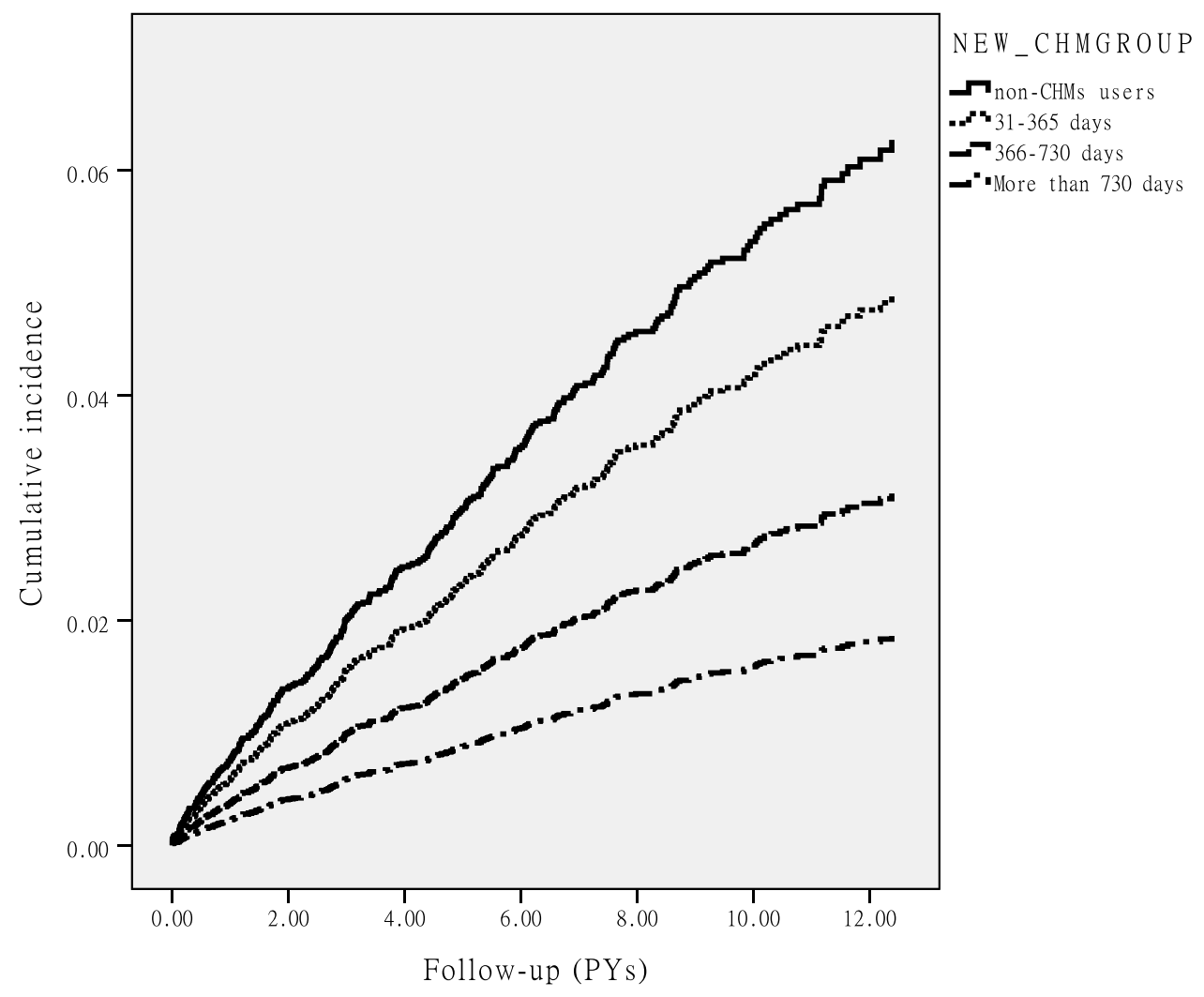

Figure 2 Cumulative incidence of COPD in RA patients with and without receiving CHMs treatment during the I5-year study period (log-rank test, $P<0.00 \mathrm{I}$ ).

proximity to their social network. ${ }^{20}$ Younger RA patients, therefore, might more easily adapt to the effects of the progression of RA, thereby diluting the therapeutic effect of CHMs. Furthermore, the reduction in risk of COPD was found to be higher for females than for males $(43 \%$ vs. $18 \%)$. We inferred that women might have superior health consciousness than men and immediately sought medical therapy at the slightest irregularity in well-being, and therefore, may be more likely to comply with the prescribed medical regimen to diminish the sequent risk of COPD. $^{21}$
An notable contribution of this work is the list of herb products that are related to the reduced risk of COPD. First, we observed the positive therapeutic effects of Yan Hu Suo and Sāng Zhī in reducing the onset of COPD. These two herbal products are typically used to relieve the level of inflammation and neuropathic pain. ${ }^{22,23}$ A murine model showed that the extract of Yan Hu Suo could suppress the production of interleukin (IL)- 6 and tumor necrosis factor- $\alpha$ (TNF- $\alpha$ ) by modulating the activation of nuclear factorkappa beta $(\mathrm{NF}-\mathrm{kB}) .{ }^{24}$ These mediators are well known to play an indispensable role in the pathogenesis of lung

Table 3 Incidence and COPD Risk for RA Patients with and Without CHMs Use, Stratified by Sex and Age

\begin{tabular}{|c|c|c|c|c|c|c|c|c|}
\hline \multirow[t]{2}{*}{ Variables } & \multicolumn{3}{|c|}{ Non- CHMs Users } & \multicolumn{3}{|c|}{ CHMs Users } & \multirow[t]{2}{*}{ Crude HR $(95 \% \mathrm{Cl})$} & \multirow[t]{2}{*}{ Adjusted HR $(95 \% \mathrm{Cl})$} \\
\hline & Case & PYs & Incidence & Case & PY & Incidence & & \\
\hline \multicolumn{9}{|l|}{ Sex } \\
\hline Female & 115 & $19,038.32$ & 6.04 & 62 & $|9,485.4|$ & 3.18 & $0.53(0.38-0.7 I)$ & $0.57^{\mathrm{Y}}(0.42-0.77)$ \\
\hline Male & 87 & 7342.32 & 11.85 & 74 & 6866.71 & 10.78 & $0.91(0.66-1.21)$ & $0.82^{\mathrm{Y}}(0.60-1.12)$ \\
\hline \multicolumn{9}{|l|}{ Age (years) } \\
\hline$\leq 50$ & 27 & $11,230.89$ & 2.40 & 20 & $11,182.57$ & 1.79 & $0.74(0.42-1.33)$ & $0.74 *(0.4 I-1.32)$ \\
\hline$>50$ & 175 & $15,149.74$ & 11.55 & 116 & $15,169.54$ & 7.65 & $0.66(0.52-0.83)$ & $0.63^{*}(0.50-0.80)$ \\
\hline
\end{tabular}

Notes: ${ }^{Y}$ Model adjusted for age, urbanization level, monthly income, medication use and comorbidities. *Model adjusted for sex, urbanization level, monthly income, medication use and comorbidities. 
Table 4 Risk of COPD in Relation to the 10 Most Used Single-Herb and Multi-Herb CHMs Products for RA Patients

\begin{tabular}{|c|c|c|c|}
\hline Chinese Herbal Product & Number of Prescriptions & Crude HR (95\% CI) & Adjusted HR* $(95 \% \mathrm{Cl})$ \\
\hline \multicolumn{4}{|l|}{ Single-herb products } \\
\hline Yan Hu Suo & 8699 & $0.45(0.34-0.62)$ & $0.57(0.42-0.70)$ \\
\hline Sāng Zhī & 2119 & $0.4 I(0.24-0.70)$ & $0.53(0.32-0.8 \mathrm{I})$ \\
\hline Chuan-Niu-Xi & 2186 & $0.67(0.43-1.04)$ & $0.79(0.47-1.14)$ \\
\hline Bei Mu & 5221 & $0.61(0.54-0.96)$ & $0.77(0.56-1.09)$ \\
\hline Du-Zhong & 5008 & $0.7 I(0.50-1.03)$ & $0.72(0.5 \mathrm{I}-\mathrm{I} .08)$ \\
\hline Dan-Shen & 5775 & $0.52(0.36-0.75)$ & $0.58(0.40-0.84)$ \\
\hline Ji-Xue-Teng & 4500 & $0.68(0.35-1.04)$ & $0.69(0.37-1.04)$ \\
\hline Huang Qin & 5230 & $0.50(0.35-0.7 I)$ & $0.59(0.45-0.90)$ \\
\hline Hai Piao Xiao & 3436 & $0.57(0.36-0.88)$ & $0.77(0.47-1.15)$ \\
\hline Da Huang & 4585 & $0.53(0.35-0.8 \mathrm{I})$ & $0.74(0.4 \mathrm{I}-1.09)$ \\
\hline \multicolumn{4}{|l|}{ Multi-herb products } \\
\hline Shu-Jing-Huo-Xue-Tang & 9390 & $0.58(0.44-0.74)$ & $0.64(0.50-0.83)$ \\
\hline Jia-Wei-Xiao-Yao-San & 8898 & $0.4 I(0.29-0.58)$ & $0.65(0.46-0.90)$ \\
\hline Shao-Yao-Gan-Cao-Tang & 5722 & $0.72(0.55-0.94)$ & $0.83(0.63-1.10)$ \\
\hline Ge-Gen-Tang & 5819 & $0.5 I(0.37-0.70)$ & $0.65(0.47-0.89)$ \\
\hline Dang-Gui-Nian-Tong-Tang & 5033 & $0.63(0.45-0.88)$ & $0.77(0.55-1.07)$ \\
\hline Xue-Fu-Zhu-Yu-Tang & 4463 & $0.68(0.50-0.94)$ & $0.76(0.57-1.06)$ \\
\hline Du-Huo-Ji-Sheng-Tang & 6623 & $0.56(0.4 I-0.77)$ & $0.57(0.42-0.77)$ \\
\hline Chuan-Xiong-Cha-Tiao-San & 4827 & $0.68(0.47-0.95)$ & $0.73(0.44-1.10)$ \\
\hline Zhi-Gan-Cao-Tang & 3677 & $0.7 I(0.48-1.07)$ & $0.77(0.52-1.12)$ \\
\hline Gan-Lu-Yin & 4101 & $0.68(0.4 I-0.94)$ & $0.75(0.45-1.06)$ \\
\hline
\end{tabular}

Notes: *Model adjusted for age, sex, urbanization level, monthly income, medication use and comorbidities.

diseases, especially COPD. ${ }^{25}$ Additionally, a recent in vivo study revealed that Sāng Zhī inhibited the lipopolysaccharide-induced production of the pro-inflammatory cytokine in the Raw264.7 cell line by blocking the "BLT2 ligandBLT2"-linked autocrine inflammatory axis, ${ }^{23}$ which may explain its therapeutic effect of decreasing the risk of COPD onset.

Another herbal product proven effective in lessening COPD risk among RA patients is Dang Shen. This remedy belongs to the Campanulaceae family. The root extracts of the Codonopsis species have been shown to possess antioxidant, anti-tumor, anti-microbial and immune-boosting properties. $^{26}$ A meta-analysis of 48 randomized controlled trials highlighted that Dang Shen exerted a number of positive effects on pulmonary function, such as improvement of forced expiratory volume (FEV1) and 6-min walking distance, compared with conventional pharmacotherapy. ${ }^{27}$ Most importantly, the integration of Codonopsis roots into routine therapy was found to significantly enhance patients' quality of life compared to placebo, as measured by St. George's Respiratory Questionnaire (standard mean difference: -7.19 , $95 \% \mathrm{CI}:-10.82$ to -3.56$).^{27}$
Huang Qin also appears to modulate the immune response, which may have important implications in chronic inflammatory diseases such as COPD. ${ }^{18,26}$ Baicalin, a major component of this formula, was proven in both in vitro and in vivo studies to have anti-inflammatory, antioxidant and antibacterial actions, as it has been shown to inhibit cytokines and transcription factors. For example, using a rodent model, Kim and colleagues discovered that Huang Qin suppressed the activation of the MEK/ERK and IKK $\alpha \beta / \mathrm{I} \kappa \mathrm{B} \alpha$-dependent pathways via c-Raf-1 activation, thereby altering the synthesis of inflammatory mediators. ${ }^{28}$

Among the commonly used multi-herb products for RA patients, we noted that Jia-Wei-Xiao-Yao-San was significantly related to a lower risk of COPD. Recent scientific evidence indicates that this formula works by increasing synaptic plasticity, upregulating the expression of hippocampal brain-derived neurotrophic factor (BDNF), ${ }^{29}$ and diminishing the level of inflammatory markers, ${ }^{30}$ which in turn decreased the subsequent COPD risk in RA patients. Elevated concentrations of both BDNF and inflammatory cytokines in the sera are well documented before a definitive diagnosis of COPD, ${ }^{31}$ implying that 
these platelet mediators are likely involved in the pathogenesis of COPD.

The positive therapeutic effects of Shu-Jing-Huo-XueTang and of Du-Huo-Ji-Sheng-Tang on the subsequent predisposition to COPD were also identified in this study. Both animal experiments and human studies have shown that the anti-inflammatory property could be extracted from their inherent compounds, such as ferulic acid and paeoniflorin from Shu-Jing-Huo-Xue-Tang, and gentianine from DuHuo-Ji-Sheng-Tang. The relevant mechanisms by which these ingredients exhibit their powerful anti-inflammatory effects may be partially associated with inhibition of the $\mathrm{NF}-\mathrm{kB}$ signaling pathway, ${ }^{32-34}$ suggesting that these formulae have the potential to treat airway inflammation diseases in addition to rheumatologic disorders.

In our study, we also discovered that the prescription of Ge-Gen-Tang was associated with lower vulnerability to COPD, echoing a previous study finding. ${ }^{35}$ It was inferred from this finding that this formula could ameliorate airway remodeling by modulating airway inflammation and oxidative stress in the lungs. Several recent studies have shown that puerarin, a major isoflavone glycoside purified from this formula, can not only help decrease the levels of IL-4 and TNF- $\alpha$ but also reduce oxidative stress in a rodent model of inflammatory disorders. ${ }^{35,36}$ A study by Huang and colleagues further indicated that these plasma inflammatory cytokines were related to measures of the severity of airway diseases, such as the FEV1/forced vital capacity ratio, and may be potential markers for the evaluation of chronic inflammatory lung diseases. ${ }^{37}$

Findings from the current study have important clinical and research implications. However, several limitations should be noted when interpreting these results. First, coding errors are always a possibility in an administrative database. To minimize this bias, we enrolled only subjects with new-onset RA or COPD, and only after the patient had at least three outpatient visits reporting consistent diagnoses or at least one inpatient admission. To verify the accuracy of medical records, the Taiwan NHI randomly samples claims from hospitals, interviews patients and reviews medical charts. ${ }^{15}$ Second, data on social network relationships, coping strategies and resources, religious beliefs or educational levels were not available from the claims files, and confounding by any of these factors may exist. Future research should include these uncontrolled variables to assess whether the present findings can be replicated across diverse groups of medical patients. Third, data regarding
RA severity were not available in this database, and failure to adjust for this factor may bias the results. However, our multivariate analysis considered the impact of multiple comorbidities, including hypertension, stroke, diabetes, heart disease, chronic kidney disease, cancer, alcohol dependence syndrome and tobacco use. Furthermore, two sensitivity analyses were also performed to further examine the relationship between CHMs use and the subsequent risk of COPD. The first sensitivity analysis, limited to RA patients with no comorbidities, found CHMs to be still protective against the development of COPD (adjusted HR $=0.53,95 \% \mathrm{CI}, 0.38-0.73)$. Second, we used the prescription of biological agents as a surrogate for RA severity, dividing subjects by whether or not they received biological agents for $\geqq 6$ months after the index date. The proportion of use of biological agents was $59.1 \%(1985 / 3360)$ in the CHMs user cohort and $55.2 \%(1858 / 3360)$ in the nonCHMs user cohort. The results of this re-analysis, that took into account the use of biological agents, were essentially the same as those reported in the original analysis (adjusted HR: 0.59, 95\% CI: 0.37-0.80). Findings from these sensitivity analyses suggest that the severity of RA did not appreciably impact the relationship reported herein. Fourth, evidence from any observational cohort study is generally less robust than that obtained from randomized trials, because cohort study designs provide little safety against existing confounding bias. Despite our meticulous efforts to control for confounding factors, unpredictable biases may remain from unmeasured or unknown confounders. Fifth, although our study revealed a substantial benefit effect of CHMs use on the reduction of COPD onset among RA patients, it must be recognized that these patients were not randomly categorized into users and nonusers. Therefore, caution should be exerted when interpreting the findings. A randomized controlled trial is, therefore, recommended to clearly determine the efficacy of these CHMs, as well as the mechanisms that underlie their successful application. These limitations notwithstanding, this study also possessed several strengths. These include the immediate availability of data, the comprehensiveness of the database and the statistical power from the use of a large, nationally representative sample. In addition, this retrospective 15-year cohort study allowed us to robustly determine the relationship between CHMs use and COPD in RA patients. Furthermore, the present findings may serve as a useful reference for future studies on this topic among other populations of patients with severe and chronic illnesses. 


\section{Conclusion}

This is the first large-scale nationwide cohort study to show the association of CHMs use with the subsequent risk of COPD among RA patients, thus suggesting a venue for further studies of the effect of CHMs on other medical conditions. We found that the integration of CHMs into the RA treatment regimen reduced the subsequent risk of COPD by $32 \%$. Results of this study may serve as a reference to help healthcare providers when planning and implementing therapeutic interventions that seek to improve the health of patients with RA.

\section{Abbreviations}

$\mathrm{RA}$, rheumatoid arthritis; COPD, chronic obstructive pulmonary disease; CHMs, Chinese herbal medicine; HR, hazard ratio; CI, Confidence Interval; LHID, Longitudinal Health Insurance Database; NHI, National Health Insurance; ICD9-CM, International Classification of Disease, Ninth Revision, Clinical Modification; PYs, person-years; NTD, New Taiwan Dollar; IL, Interleukin; TNF- $\alpha$, tumor necrosis factor- $\alpha$; NF$\kappa \mathrm{B}$, nuclear factor-kappa beta; FEV1, forced expiratory volume; BDNF, brain-derived neurotrophic factor.

\section{Acknowledgments}

The study is based in part on data from the National Health Insurance Research Database provided by the Bureau of National Health Insurance, Department of Health and managed by the National Health Research Institutes, Taiwan. The interpretation and conclusions contained herein do not represent those of the Bureau of National Health Insurance, Department of Health or National Health Research Institutes.

\section{Author Contributions}

All authors contributed to data analysis, drafting and revising the article, gave final approval of the version to be published, and agree to be accountable for all aspects of the work.

\section{Disclosure}

The authors report no conflicts of interest in this work.

\section{References}

1. Woolf AD, Pfleger B. Burden of major musculoskeletal conditions. Bull World Health Organ. 2003;81(9):646-656.

2. Allaire S, Wolfe F, Niu J, Lavalley MP. Contemporary prevalence and incidence of work disability associated with rheumatoid arthritis in the US. Arthritis Rheum. 2008;59(4):474 480. doi:10.1002/(ISSN)1529-0131
3. Chen CI, Wang L, Wei W, Yuce H, Phillips K. Burden of rheumatoid arthritis among US medicare population: co-morbidities, health-care resource utilization and costs. Rheumatol Adv Pract. 2018;2(1):I1-I9.

4. Birnbaum H, Pike C, Kaufman R, Maynchenko M, Kidolezi Y, Cifaldi M. Societal cost of rheumatoid arthritis patients in the US. Curr Med Res Opin. 2010;26(1):77-90. doi:10.1185/03007990903422307

5. Mcguire K, Aviña-Zubieta JA, Esdaile JM, et al. Risk of incident chronic obstructive pulmonary disease in rheumatoid arthritis: a population-based cohort study. Arthritis Care Res. 2019;71 (5):602-610. doi:10.1002/acr.23410

6. Ungprasert P, Srivali N, Cheungpasitporn W, Davis IJ. Risk of incident chronic obstructive pulmonary disease in patients with rheumatoid arthritis: a systematic review and meta-analysis. Joint Bone Spine. 2016;83(3):290-294. doi:10.1016/j.jbspin.2015.05.016

7. Hyldgaard C, Bendstrup E, Pedersen AB, et al. Increased mortality among patients with rheumatoid arthritis and COPD: a population-based study. Respir Med. 2018;140:101-107. doi:10.1016/j.rmed.2018.06.010

8. Lassere MN, Rappo J, Portek IJ, Sturgess A, Edmonds JP. How many life years are lost in patients with rheumatoid arthritis? Secular cause-specific and all-cause mortality in rheumatoid arthritis, and their predictors in a long-term Australian cohort study. Intern Med J. 2013;43(1):66-72. doi:10.1111/j.1445-5994.2012.02727.x

9. Barnes PM, Bloom B, Nahin RL. Complementary and alternative medicine use among adults and children: United States, 2007. Natl Health Stat Report. 2008;10(12):1-23.

10. Chang LC, Huang N, Chou YJ, Lee CH, Kao FY, Huang YT. Utilization patterns of Chinese medicine and Western medicine under the national health insurance program in Taiwan, a population -based study from 1997to 2003. BMC Health Serv Res. 2008;8:170. doi:10.1186/14726963-8-170

11. Lai JN, Wu CT, Wang JD. Prescription pattern of Chinese herbal products for breast cancer in Taiwan: a population-based study. Evid Based Complement Alternat Med. 2012;2012:891893. doi:10.1155/ 2012/891893

12. Liao YN, Hu WL, Chen HJ, Hung YC. The use of Chinese herbal medicine in the treatment of chronic obstructive pulmonary disease (COPD). Am J Chin Med. 2017;45(2):225-238. doi:10.1142/ S0192415X17500148

13. Lee YW, Chen TL, Shih YR, et al. Adjunctive traditional Chinese medicine therapy improves survival in patients with advanced breast cancer: a population-based study. Cancer. 2014;120(9):1338-1344. doi:10.1002/cncr.28579

14. Li JS, Li SY, Xie Y, et al. The effective evaluation on symptoms and quality of life of chronic obstructive pulmonary disease patients treated by comprehensive therapy based on traditional Chinese medicine patterns. Complement Med. 2013;21(6):595-602. doi:10.1016/j. ctim.2013.09.006

15. National Health Insurance Research Database, Taiwan. Available from: http://nhird.nhri.org.tw/date_cohort.html. Accessed January 18, 2019.

16. Tsai TY, Livneh H, Hung TH, Lin IH, Lu MC, Yeh CC. Associations between prescribed Chinese herbal medicine and risk of hepatocellular carcinoma in patients with chronic hepatitis B: a nationwide population-based cohort study. BMJ Open. 2017;7(1):e014571. doi:10.1136/bmjopen-2016-014571

17. Liu CY, Hung YT, Chuang YL, et al. Incorporating development stratification of Taiwan townships into sampling design of large scale health interview survey. J Health Manag. 2006;4(1):1-22.

18. Coyle M, Shergis JL, Liu S, et al. Safety of Chinese herbal medicine for chronic obstructive pulmonary disease. Evid Based Complement Alternat Med. 2015;2015:12. doi:10.1155/2015/380678

19. Siddiqui MJ, Min CS, Verma RK, Jamshed SQ. Role of complementary and alternative medicine in geriatric care: a mini review. Pharmacogn Rev. 2014;8(16):81-87. doi:10.4103/0973-7847.134230

20. Cornwell B, Laumann EO, Schumm LP. The social connectedness of older adults: a national profile. Am Sociol Rev. 2008;73(2):185-203. doi:10.1177/000312240807300201 
21. Shih CC, Liao CC, Su YC, Tsai CC, Lin JG. Gender differences in traditional Chinese medicine use among adults in Taiwan. PLoS One. 2012;7(4):e32540. doi:10.1371/journal.pone.0032540

22. Wang L, Zhang Y, Wang Z, et al. The antinociceptive properties of the corydalis yanhusuo extract. PLoS One. 2016;11(9):e0162875. doi:10.1371/journal.pone.0162875

23. Park GS, Kim JK, Kim JH. Anti-inflammatory action of ethanolic extract of ramulus mori on the BLT2-linked cascade. BMB Rep. 2016;49(4):232-237. doi:10.5483/BMBRep.2016.49.4.002

24. Li W, Huang H, Zhang Y, et al. Anti-inflammatory effect of tetrahydrocoptisine from corydalis impatiens is a function of possible inhibition of TNF- $\alpha$, IL-6 and NO production in lipopolysaccharidestimulated peritoneal macrophages through inhibiting NF- $\kappa \mathrm{B}$ activation and MAPK pathway. Eur J Pharmacol. 2013;715(1-3):62-71. doi:10.1016/j.ejphar.2013.06.017

25. Tian Y, Li Y, Li J, et al. Bufei yishen granules combined with acupoint sticking therapy suppress inflammation in chronic obstructive pulmonary disease rats: via JNK/p38 signaling pathway. Evid Based Complement Alternat Med. 2017;2017:1768243. doi:10.1155/2017/1768243

26. Chen HY, Ma $\mathrm{CH}$, Cao $\mathrm{KJ}$, et al. A systematic review and meta-analysis of herbal medicine on chronic obstructive pulmonary diseases. Evid Based Complement Alternat Med. 2014;2014:925069. doi:10.1155/2014/726740

27. Shergis JL, Liu S, Chen X, et al. Dang shen [codonopsis pilosula (Franch.) Nannf] herbal formulae for chronic obstructive pulmonary disease: a systematic review and meta-analysis. Phytother Res. 2015;29(2):167-186. doi:10.1002/ptr.5248

28. Kim EH, Shim B, Kang S, et al. Anti-inflammatory effects of scutellaria baicalensis extract via suppression of immune modulators and MAP kinase signaling molecules. J Ethnopharmacol. 2009;126 (2):320-331. doi:10.1016/j.jep.2009.08.027

29. Shen J, Zhang J, Deng M, Liu Y, Hu Y, Zhang L. The antidepressant effect of angelica sinensis extracts on chronic unpredictable mild stress-induced depression is mediated via the upregulation of the BDNF signaling pathway in rats. Evid Based Complement Alternat Med. 2016;2016:7434692. doi:10.1155/2016/7434692
30. Chiu CT, Chuang CY, Li JR, Huang HY, Chang SW, Hung YC. Greater therapeutic efficacy of prednisolone plus medicinal herbs than prednisolone or medicinal herbs alone in patients with oral lichen planus. J Dent Sci. 2010;5(4):209-215. doi:10.1016/j. jds.2010.11.004

31. Stoll P, Wuertemberger U, Bratke K, Zingler C, Virchow JC, Lommatzsch M. Stage-dependent association of BDNF and TGF- $\beta 1$ with lung function in stable COPD. Respir Res. 2012;13(1):116. doi:10.1186/1465-9921-13-116

32. Guo RB, Wang GF, Zhao AP, Gu J, Sun XL, Hu G. Paeoniflorin protects against ischemia-induced brain damages in rats via inhibiting MAPKs/NF- $\mathrm{BB}$-mediated inflammatory responses. PLoS One. 2012;7 (11):e49701. doi:10.1371/journal.pone.0049701

33. Kim JK, Kwon YI, Jang HD. Anti-inflammatory effects of catechol and ferulic acid derivatives through NF- $\kappa \mathrm{B}$ activation in Raw264.7 cells (830.21). FASEB J. 2014;28(1):830.

34. Wenjin C, Jianwei W. Protective effect of gentianine, a compound from Du Huo Ji Sheng Tang, against freund's complete adjuvantinduced arthritis in rats. Inflammation. 2017;40(4):1401-1408. doi:10.1007/s10753-017-0583-8

35. Dong F, Wang C, Duan J, Zhang W, Xiang D, Li M. Puerarin attenuates ovalbumin-induced lung inflammation and hemostatic unbalance in rat asthma model. Evid Based Complement Alternat Med. 2014;2014:726740.

36. Yang X, Zhang H, Wang J, Zhang Z, Li C. Puerarin decreases bone loss and collagen destruction in rats with ligature-induced periodontitis. J Periodontal Res. 2015;50(6):748-757. doi:10.1111/ jre.2015.50.issue-6

37. Huang AX, Lu LW, Liu WJ, Huang M. Plasma inflammatory cytokine IL-4, IL-8, IL-10, and TNF- $\alpha$ levels correlate with pulmonary function in patients with asthma-chronic obstructive pulmonary disease (COPD) overlap syndrome. Med Sci Monit. 2016;22:2800-2808. doi:10.12659/MSM.896458

\section{Publish your work in this journal}

The International Journal of COPD is an international, peer-reviewed journal of therapeutics and pharmacology focusing on concise rapid reporting of clinical studies and reviews in COPD. Special focus is given to the pathophysiological processes underlying the disease, intervention programs, patient focused education, and self management protocols. This journal is indexed on PubMed Central, MedLine and CAS. The manuscript management system is completely online and includes a very quick and fair peer-review system, which is all easy to use. Visit http://www.dovepress.com/testimonials.php to read real quotes from published authors. 